\title{
El funcionamiento cognitivo como endofenotipo de la anorexia nerviosa. Estudio en familiares no afectados por la enfermedad
}

\section{Cognitive functioning as an endophenotype of anorexia nervosa.}

\author{
A study in first-degree relatives unaffected by the disease
}

Fernanda Tapajóz ${ }^{1}$, Sebastián Soneira ${ }^{2}$ y Ricardo F. Allegri ${ }^{3}$

\begin{abstract}
${ }^{1}$ Doctora en Psicología de la Universidad de Buenos Aires. Especialista en Neuropsicología Clínica (Universidad de Buenos Aires - UBA). Miembro del Servicio de Neurología Cognitiva, Neuropsicología y Neuropsiquiatría de la Fundación para la Lucha contra las Enfermedades

Neurológicas de la Infancia (FLENI). Becaria posdoctoral del Consejo Nacional de Investigaciones Científicas y Técnicas (CONICET). E-mail: fetapajoz@hotmail.com

${ }^{2}$ Médico especialista en Psiquiatría. Miembro activo de la Academy for Eating Disorders y presidente del capítulo internacional de dicha academia. Responsable de la sección de Trastornos Alimentarios y Psiquiatría Nutricional del Servicio de Psiquiatría de FLENI.

E-mail: sebastiansoneira@gmail.com

${ }^{3}$ Médico especialista en Neurología y Psiquiatría. Jefe del Servicio de Neurología Cognitiva, Neuropsicología y Neuropsiquiatría de FLENI. Profesor de Neurología de la Facultad de Medicina de la Universidad de Buenos Aires y de Neurociencias de la Universidad de la Costa (Colombia). Fellow de la Academia Americana de Neurología y miembro del Consejo Mundial de Demencia. E-mail: rallegri@fleni.org.ar
\end{abstract}

La doctora Tapajoz agradece a sus colegas del Servicio de Neurología Cognitiva de FLENI. Los autores agradecen a la Clínica de Nutrición Dr. Cormillot, al Hospital Argerich y a todos los participantes involucrados en el estudio.

Servicio de Neurología Cognitiva, Neuropsicología y Neuropsiquiatría, FLENI. Buenos Aires, Argentina.

\section{Resumen}

El perfil cognitivo de los pacientes con anorexia nerviosa se caracteriza por dificultades en la flexibilidad mental y en la coherencia central. El objetivo de este trabajo fue analizar si los familiares de primer grado no afectados de los pacientes presentan estas dificultades cognitivas, que podrían representar rasgos endofenotípicos de la enfermedad.

Fueron estudiadas 34 mujeres: 17 familiares de primer grado (madres y hermanas) de pacientes con anorexia nerviosa y 17 controles sanos agrupados por edad y escolaridad. Se consideraron el índice de masa corporal, la ansiedad, la depresión, los síntomas obsesivo-compulsivos y los relacionados con los trastornos alimentarios. Se evaluó la coherencia central, mediante la copia de la Figura Compleja de Rey, y la flexibilidad mental, mediante el test de Stroop, el test de los trazos $B$ y el test de fluencia fonológica.

Los familiares de pacientes con anorexia nerviosa presentaron un menor rendimiento en las medidas de coherencia central $(\mathrm{p}<$ $.05)$ y en fluencia fonológica $(\mathrm{p}<.05)$ que los controles sanos. Se observó una correlación entre el test de Stroop y los síntomas de depre- 
sión y trastornos alimentarios $(\mathrm{p}<.05)$.

Los familiares de primer grado no afectados de pacientes con anorexia nerviosa presentaron dificultades en la coherencia central y, en menor grado, en la flexibilidad mental. Los resultados en los familiares indican que este perfil podría ser mediado genéticamente, constituyendo un rasgo característico de la anorexia nerviosa $\mathrm{y}$, por ende, un posible candidato a endofenotipo neuropsicológico de esta enfermedad.

Palabras clave: anorexia nerviosa, neuropsicología, coherencia central, flexibilidad mental, endofenotipo.

\section{Abstract}

The cognitive profile of patients with anorexia nervosa is characterized by difficulties in central coherence and mental flexibility. Central coherence is defined by the ability to integrate incoming information in its own context, and weakness in central coherence is characterized by poor overall processing and superior detail processing. Mental flexibility is defined by the ability to change the course of a thought or action according to the demands of the environment. Alterations in this cognitive domain generate rigid and inflexible behavior. An open question in the literature is whether these cognitive characteristics are a transient state derived from the disease or whether they are stable traits associated with anorexia nervosa and endophenotypical features of this disease. The concept of endophenotype refers to the internal phenotype that is not clinically appreciable but can be observed indirectly through deficits that arise in the performance of certain neuropsychological tests. In recent years the search for endophenotypes has been renewed in the field of psychiatry as they would constitute an important route for the understanding of the biological and genetic bases of mental illnesses, constituting markers that allow a diagnosis before the onset of clinical symptomatology. For a cognitive marker to be considered an endophenotype it must meet a series of characteristics such as being measurable, inherited, found in patients with and without active disease and in first-degree relatives not affected by the disease. The aim of the present study was to assess whether difficulties in central coherence and mental flexibility are shared by unaffected first-degree relatives of patients with anorexia nervosa and thus constitute an endophenotypical feature of this disease.

This is a cross-sectional, descriptive-comparative study in which 34 women participated: 17 unaffected first-degree relatives of patients with anorexia nervosa (mothers and sisters) and 17 healthy controls matched by age and education. For the study of central coherence the copy of Rey's Complex Figure was used and to assess set-shifting the Stroop test, the Trail Making Test B and the Phonological Fluency test were used. Demographic and clinical aspects such as age, educational level, body mass index, anxiety, depression, obsessive-compulsive and eating disorder related symptoms were also evaluated.

First-degree relatives of patients with anorexia nervosa showed lower performance on measures of central coherence $(p<.05)$ and phonological fluency $(p<.05)$ than healthy controls. A correlation was observed between the Stroop test and depression and eating disorders symptoms $(\mathrm{p}<.05)$.

The results of this study show that unaffected first-degree relatives of patients with anorexia nervosa presented alterations in central coherence and, to a lesser degree, in mental flexibility. These results, in addition to previous research in which difficulties persisted even after recovery, indicate that these alterations could be genetically mediated, constituting a characteristic trait of anorexia nervosa and therefore a possible candidate for neuropsychological endophenotype of this disease. Regarding practical implications of the study, the findings reinforce the importance of cognitive remediation treatments not only for patients with anorexia nervosa but also emphasize that they could be 
useful for unaffected family members. Taking into account that family intervention is a widely used tool in the psychological treatment of anorexia, improving the perception of the patient and his relatives about cognitive biases, could contribute to raising awareness of the disease, something that patients with anorexia nervosa do not usually have, and generate a positive impact on the response to treatment as a whole.

Keywords: anorexia nervosa, neuropsychology, central coherence, mental flexibility, endophenotype.

\section{Introducción}

La anorexia nerviosa (AN) es un trastorno psiquiátrico grave que presenta la tasa de mortalidad más alta de todas las enfermedades psiquiátricas (Arcelus, Mitchell, Wales, y Nielsen, 2011). Se define por la pérdida de peso inducida por la restricción alimentaria, asociada con la alteración de la imagen corporal y el miedo mórbido al aumento de peso, lo que produce graves disfunciones físicas, conductuales, cognitivas y emocionales (American Psychiatric Association [APA], 2013). Tiene su inicio con mayor frecuencia durante la adolescencia (Herpertz-Dahlmann, 2015) y, aproximadamente, la mitad de los individuos afectados desarrollan un curso crónico de la enfermedad, lo que genera un elevado grado de discapacidad en mujeres jóvenes en una etapa de la vida altamente productiva, con gran perjuicio en la calidad de vida (Lock et al., 2010). Hasta la fecha, la etiología de la AN no está adecuadamente determinada; sin embargo, un consenso creciente sugiere un origen multifactorial, en el cual los factores neuropsicológicos y neurobiológicos pueden contribuir a la vulnerabilidad, el inicio y el mantenimiento de la enfermedad (Kaye, Wierenga, Bailer, Simmons y BischoffGrethe, 2013; Treasure et al., 2015). Las altas tasas de falta de efectividad de los tratamientos (Waller et al., 2014), la mortalidad alarmantemente alta (Arcelus et al., 2011) y los graves riesgos médicos y psíquicos asociados con la AN determinan la necesidad de reconocer este trastorno lo más precozmente posible e intervenir con el tratamiento actualmente disponible para minimizar las consecuencias de la enfermedad.

En las últimas décadas se ha observado un notable incremento de estudios centrados en los aspectos neuropsicológicos y neurobiológicos de la AN. El perfil cognitivo de los pacientes ha recibido particular atención y, actualmente, está cada vez más reconocido que los individuos con esta patología presentan importantes dificultades en el funcionamiento neuropsicológico (Jáuregui-lobera, 2013). Las características cognitivas más consensuadas en la literatura son la debilidad en la coherencia central -habilidad de integrar la información entrante en su contexto; la debilidad en la coherencia central que se caracteriza por un procesamiento global pobre y un superior procesamiento del detalle- (López, Tchanturia, Stahl y Treasure, 2008; López et al., 2008; Tapajóz et al., 2016) y las alteraciones en el funcionamiento ejecutivo, particularmente en la flexibilidad mental - capacidad de cambiar el curso de un pensamiento o acción según las demandas del ambiente- (Lounes, Khan y Tchanturia, 2011; Roberts, Tchanturia, Stahl, Southgate y Treasure, 2007; Tchanturia et al., 2011; Tchanturia et al., 2012).

Una cuestión debatida es si estas características cognitivas constituyen una consecuencia de la enfermedad, como el producto del estado crónico de privación nutricional en la estructura y funcionamiento cerebral, o si podrían ser rasgos endofenotípicos de la AN. En los últimos años se ha renovado la búsqueda de endofenotipos para las enfermedades psiquiátricas, ya que configurarían una importante vía para la comprensión de las bases biológicas y genéticas de las enfermedades, pudiendo ser marcadores que permitan hacer un diagnóstico que preceda a la sintomatología clínica y a su curso. El concepto de "endofenotipo" se refiere al fenotipo interno que clínicamente no es apreciable, pero que 
puede observarse de manera indirecta a través de los déficits que surgen en la ejecución de determinadas pruebas neuropsicológicas. Para que un marcador cognitivo sea considerado un endofenotipo debe reunir una serie de características (Gottesman y Gould, 2003; Miranda, Jaramillo, Valencia y Duque, 2003) como ser medible, hereditario, encontrarse en los pacientes con y sin enfermedad activa y en familiares de primer grado no afectados por la enfermedad.

La búsqueda de endofenotipos cognitivos es particularmente importante en el caso de la anorexia nerviosa considerando su elevada tasa de mortalidad así como de ineficiencia de los tratamientos disponibles (Agras et al., 2004; Fairburn et al., 2009; Pike, 1998). En este sentido, identificar endofenotipos para la AN es de relevancia porque, además de encontrar factores de vulnerabilidad a la enfermedad, podría contribuirse al desarrollo de nuevos tratamientos, así como a la construcción de modelos etiológicos (Treasure, 2007).

Los déficits en las funciones ejecutivas y la coherencia central han sido considerados por algunos autores como posibles candidatos a endofenotipos para esta enfermedad, ya que fueron encontrados en pacientes recuperados $\mathrm{y}$ en familiares de primer grado no afectados por la enfermedad (Galimberti et al., 2013; Harrison, Tchanturia, Naumann y Treasure, 2012; Holliday, Tchanturia, Landau, Collier y Treasure, 2005; Kanakam, Raoult, Collier y Treasure, 2013; Lang, Treasure y Tchanturia, 2015b; Lopez, Tchanturia, Stahl y Treasure, 2009; Tenconi et al., 2010). Sin embargo, otros trabajos presentaron resultados divergentes. Talbot, Hay, Buckett y Touyz (2015) no encontraron diferencias significativas entre individuos con anorexia nerviosa aguda, recuperados de la AN y controles sanos (CS) en medidas de coherencia central. Se observaron, únicamente, diferencias en la flexibilidad mental entre los individuos recuperados y los CS y, sorprendentemente, no las encontraron entre los pacientes con AN aguda y los CS. Por otro lado, Brown et al. (2018) no observaron diferencias significativas en la flexibilidad mental en pacientes jóvenes con AN, y encontraron parcial evidencia de alteraciones en la coherencia central. Asimismo, no encontraron diferencias entre el desempeño de los familiares y los CS. En una reciente revisión que buscó evaluar si los déficits en la flexibilidad mental y coherencia central persisten en individuos recuperados de la AN, Fuglset (2019) observó que las alteraciones en la flexibilidad mental persisten en los individuos recuperados. Por otro lado, en cuanto a la coherencia central, concluyó que los resultados son aún inconsistentes.

La discrepancia observada entre los estudios mencionados indica que son necesarias más investigaciones sobre el tema. La mayor parte de los estudios referidos fue realizada con pacientes recuperados, por lo que son necesarios más estudios que evalúen a los familiares no afectados para poder evaluar posibles endofenotipos. En Argentina no existen trabajos de investigación que hayan explorado los endofenotipos cognitivos para la anorexia nerviosa.

El objetivo principal del presente estudio fue evaluar la coherencia central y la flexibilidad mental en los familiares de primer grado no afectados de pacientes con anorexia nerviosa (F-AN) en comparación con un grupo control. La hipótesis del trabajo fue que dichos familiares presentarían un perfil cognitivo similar al observado en los pacientes, según lo reportado en estudios previos (Lang, Lopez, Stahl, Tchanturia y Treasure, 2014; Lopez et al., 2008; Roberts et al., 2007; Tchanturia et al., 2012.

\section{Materiales y métodos}

\section{Participantes}

En el presente estudio participaron 17 familiares de primer grado no afectados (ocho madres y nueve hermanas) de pacientes diagnosticados con anorexia nerviosa según los 
criterios del DSM-5 (American Psychiatric Association, 2013), que reciben tratamiento en la Clínica de Nutrición Dr. Cormillot y en el Hospital Argerich de Buenos Aires (Argentina) y 17 controles sanos agrupados por edad y escolaridad.

Los criterios de exclusión fueron: (1) presencia de un trastorno psiquiátrico actual y/o antecedentes de enfermedades neurológicas, psiquiátricas o sistémicas que pudieran afectar el rendimiento cognitivo; (2) uso de medicamentos que alteren la cognición; (3) abuso de alcohol y/o sustancias ilegales; (4) presencia de algún trastorno alimentario evaluada a través del EDI-II; (5) embarazo y/o lactancia; y (6) un índice de masa corporal (IMC) menor a 18.5.

Los controles sanos fueron derivados a través de diversas fuentes, como personal administrativo de las instituciones involucradas en el estudio, colegas universitarios y miembros de la comunidad local.

\section{Procedimiento y consideraciones éticas}

Se utilizó un diseño descriptivo-analítico de corte transversal para evaluar el desempeño cognitivo en los familiares de primer grado no afectados de pacientes con AN.

Antes de la evaluación, se realizó una entrevista con todos los participantes para explicar el propósito del estudio, aclarar posibles dudas y firmar el consentimiento informado, que contaba con la aprobación del comité de ética institucional. La caracterización de los familiares y la no ocurrencia de alguno de los criterios de exclusión fueron determinadas por el segundo autor, médico psiquiatra especializado en trastornos alimentarios. La evaluación cognitiva de los participantes fue realizada por la primera autora, psicóloga especializada en Neuropsicología Clínica, en un ambiente tranquilo, libre de estímulos externos, e incluyó un estudio exhaustivo tanto neuropsicológico como clínico-psicopatológico. A los fines de este estudio, solo se informaron los resultados de la evaluación de la flexibilidad mental y coherencia central, y la evaluación clínico-psicopatológica.

\section{Medidas}

\section{Cribado cognitivo}

El Mini Mental State Examination (MMSE) (Butman et al., 2001; Folstein, Folstein y McHugh, 1975) consiste en un test de rastreo cognitivo breve compuesto por 30 ítems que evalúan la orientación en el tiempo y espacio, la codificación, la atención y concentración, el recuerdo, el lenguaje y la viso-construcción.

\section{Evaluación clínico-psicopatológica}

El Inventario de Depresión de Beck (BDI) (Beck, Steer y Brown, 2006; Brenlla y Rodríguez, 2006) consiste en 21 ítems que evalúan la existencia y severidad de síntomas de depresión. Según la investigación psicométrica de los autores, el instrumento tiene adecuadas propiedades psicométricas (coeficiente alfa de .88 para los pacientes y .86 para los individuos del grupo control).

El Inventario de Ansiedad Estado-Rasgo (STAI) (Leibovich de Figueroa, 1991; Spielberger, Gorsuc y Lushene, 1982) consiste en 40 ítems, de los cuales 20 evalúan la ansiedad como un estado transitorio y los otros 20 , la ansiedad como un rasgo estable. La adaptación argentina del inventario STAI ha demostrado adecuadas propiedades psicométricas tanto para uso clínico como para investigación $(\alpha=.90)$.

El Inventario de Obsesiones y Compulsiones - Revisado (OCI-R) (Foa et al., 2002; Fullana et al., 2005) es una encuesta tipo Likert de 18 ítems que evalúa los síntomas asociados al trastorno obsesivo compulsivo en seis dimensiones: comprobación (por ejemplo, comprobar repetidamente puertas, ventanas, etcétera); acumulación (por ejemplo, acumular cosas que no necesita); neutralización (por ejemplo, sentir que existen números buenos y malos, sentir la necesidad de contar mien- 
tras se está realizando otra tarea); obsesiones (por ejemplo, no poder controlar sus propios pensamientos); orden (por ejemplo, la necesidad de que los objetos estén bien ordenados) y lavado (por ejemplo, lavarse las manos más de lo necesario). La consistencia interna para la escala completa del OCI-R es adecuada ( $\alpha$ $=.86)$.

El Inventario de Trastornos Alimentarios II (EDI II) (Garner, 1998) evalúa la presencia de síntomas asociados a los trastornos alimentarios agrupados en 11 subescalas (Búsqueda de delgadez, Bulimia, Insatisfacción con la imagen corporal, Ineficacia, Perfeccionismo, Desconfianza interpersonal, Conciencia interoceptiva, Miedo a madurar, Ascetismo, Impulsividad e Inseguridad social). Para los propósitos de este trabajo se reportan solamente los resultados de las tres subescalas de riesgo (Rutsztein, Murawski, Elizathe y Scappatura, 2010): Búsqueda de delgadez, Bulimia e Insatisfacción con la imagen corporal y la Puntuación total. En relación con la consistencia interna del instrumento, la fiabilidad evaluada a través del alfa de Cronbach oscila entre .83 y .93 en las distintas categorías.

\section{Evaluación de coherencia central y flexibilidad mental}

\section{Coherencia central.}

Estrategia de Copia de la Figura Compleja de Rey -FCR- (análisis cuantitativo) (Booth, 2006; Lopez et al., 2008; Rey, 2003), para evaluar la coherencia central (CC) que permite un análisis cuantitativo de la estrategia de copia y arroja tres índices de coherencia central: (1) Índice Orden de Construcción (IOC) (rango: 0 - 3.3); (2) Índice Estilo (IE) (rango: 0 - 2); y (3) Índice Coherencia Central (ICC) (rango: 0 -2). Para más detalles acerca de la puntuación y administración, ver Tapajóz P. de Sampaio et al. (2013).

\section{Flexibilidad mental.}

Fluencia Verbal Fonológica (Benton, 1968; Butman, Allegri, Harris y Drake, 2000) es aquella en la que el participante debe nombrar la mayor cantidad de palabras posible que empiecen con la letra " $p$ " en un minuto. El puntaje se obtiene por el número de palabras nombradas.

Test de los trazos B (TMTB, del inglés Trail Making Test B) (Fernandéz, Marino y Alderete, 2002; Reitan, 1958), que consiste en una hoja en la cual se encuentran distribuidos al azar y de forma irregular números (del 1 al 13) y letras (de la "A" a la "L"). El participante debe unir los estímulos alternando entre los números y las letras, respetando el orden numérico ascendente y alfabético. El puntaje es el tiempo total (expresado en segundos) que le lleva al individuo realizar la tarea.

Test de Stroop (Golden, 1964) es una prueba que consiste en tres láminas con 100 elementos cada una distribuidos en cinco columnas de 20 elementos. La primera lámina (Lectura Palabras - P) está compuesta por nombres de colores escritos en tinta negra que el participante debe leer en 45 segundos. La segunda lámina (Lectura Color - C) consiste en colores de tinta que el sujeto debe nombrar en 45 segundos $y$, finalmente, la tercera lámina (Lectura Color de la Palabra - C/P) consiste en nombres de colores impresos con una tinta distinta al color que le corresponde, en la que el paciente también debe leer en 45 segundos. En esta tercera etapa del test, el participante debe inhibir la respuesta automática de la lectura a favor de una tarea novedosa que requiere una respuesta controlada. La puntuación bruta consiste en el número total de elementos leído en cada ensayo. Tras la medición de estos tres puntajes brutos $(\mathrm{P}, \mathrm{C} \mathrm{y}$ $\mathrm{C} / \mathrm{P})$, se realizan algunos cálculos a posteriori, con la finalidad de obtener el puntaje de interferencia. Con las dos primeras medidas, $\mathrm{Py} \mathrm{C}$, se calcula PC1, una estimación de la puntuación que el sujeto debería obtener en la condición de interferencia $(\mathrm{PC})$.

$$
\mathbf{P C 1}=(\mathbf{P} \times \mathbf{C}) /(\mathbf{P}+\mathbf{C})
$$

Posteriormente, la resta entre la puntuación que realmente obtiene en la condición de interferencia (PC) y la estimación de la que debería obtener (PC1) es el indicador que informa de 
cuánto el individuo se deja interferir por el efecto Stroop.

\section{INTERFERENCIA $=$ PC - PC1}

La fiabilidad del test de Stroop se ha mostrado consistente, con valores de $.86, .82$ y .73 en la aplicación individual (Golden, 1964).

\section{Análisis estadístico}

Los datos fueron analizados con el paquete estadístico para las ciencias sociales (SPSS), versión 19 para Windows. Todas las variables del estudio fueron evaluadas respecto a la normalidad de distribución y homogeneidad de la varianza a través del test de Shapiro-Wilk y del test de Levene, respectivamente. Los supuestos paramétricos no se cumplieron para la mayoría de las variables estudiadas; por esta razón se decidió utilizar la prueba no paramétrica $U$ de Mann-Whitney para la comparación entre los grupos. Para explorar la relación entre el desempeño cognitivo y las variables clínicas y psicopatológicas en el grupo F-AN se utilizó el coeficiente de correlación de Spearman $(\rho)$. Todas las pruebas se llevaron a cabo al nivel del $5 \%$ de significancia estadística.

\section{Resultados}

\section{Datos clínicos y demográficos}

En la tabla 1 se presentan las características clínicas y demográficas de los participantes.

Los grupos fueron comparables en edad, escolaridad, IMC y funcionamiento cognitivo global (por Mini-Mental State Examination). No se observaron diferencias estadísticamente significativas en los demás parámetros clínicos.

Tabla 1

Datos clínicos y demográficos de los participantes.

\begin{tabular}{c|c|c|c|c} 
& Familiares AN & $\mathbf{C S}$ & & \\
\hline & $\mathbf{( n = 1 7 )}$ & $\mathbf{( n = 1 7 )}$ & Estadístico U & $\mathbf{p}$ \\
\cline { 2 - 5 } Edad & $\mathbf{M}(\mathbf{D E})$ & $\mathbf{M}(\mathbf{D E})$ & 131.5 & $\mathrm{~ns}$ \\
Años de escolaridad & $14.4(2.2)$ & $15.7(2.1)$ & 103.0 & $\mathrm{~ns}$ \\
Índice de masa corporal & $22.4(3.6)$ & $22.9(2.5)$ & 110.0 & $\mathrm{~ns}$ \\
$\left(\mathrm{Kg} / \mathrm{m}^{2}\right)$ & $29.5(0.6)$ & $29.6(0.4)$ & 133.0 & $\mathrm{~ns}$ \\
Mini mental & $9.6(7.3)$ & $5.7(5.8)$ & 93.5 & $\mathrm{~ns}$ \\
BDI & $23.2(12.4)$ & $15.6(9.2)$ & 84.0 & $\mathrm{~ns}$ \\
STAI- Estado & $24.4(10.4)$ & $21.0(9.7)$ & 103.0 & $\mathrm{~ns}$ \\
STAI- Rasgo & $15.0(8.2)$ & $15.6(9.8)$ & 126.5 & $\mathrm{~ns}$ \\
OCI-R Total & $1.8(3.7)$ & $1.4(2.1)$ & 133.5 & $\mathrm{~ns}$ \\
EDI-II Impulso delgadez & $0.0(0.0)$ & $0.1(0.4)$ & 128.0 & $\mathrm{~ns}$ \\
EDI-II Bulimia & $3.3(3.8)$ & $4.5(4.6)$ & 120.0 & $\mathrm{~ns}$ \\
EDI-II Insatisfacción & $34.3(12.2)$ & $25.0(15.4)$ & 83.5 & $\mathrm{~ns}$ \\
corporal &
\end{tabular}

Nota: AN: anorexia nerviosa; CS: Controles sanos; BDI: Inventario de Depresión de Beck; STAI: Inventario de Ansiedad Estado-Rasgo; OCI-R: Inventario de Obsesiones y Compulsiones - Revisado; EDI-II: Inventario de Trastornos Alimentarios; ns: no significativo. 


\section{Desempeño cognitivo}

En la tabla 2 se presentan los resultados del desempeño en las tareas de coherencia central y flexibilidad mental.

\section{Coherencia central}

Se observó que el grupo de los familiares de los pacientes con AN presentó un menor rendimiento en todos los índices de coherencia central en comparación con los CS ( $\mathrm{p}<.05)$.

\section{Flexibilidad mental}

Únicamente se observaron diferencias entre los grupos en la tarea de fluencia fonológica, a favor de los CS $(\mathrm{p}<.05)$.

Tabla 2

Desempeño evaluación coherencia central y flexibilidad mental.

\begin{tabular}{c|c|c|c|c} 
& $\begin{array}{c}\text { Familiares AN } \\
(\mathbf{n}=\mathbf{1 7}) \\
\mathbf{M}(\mathbf{D E})\end{array}$ & $\begin{array}{c}\mathbf{C S} \\
(\mathbf{n}=\mathbf{1 7}) \\
\mathbf{M}(\mathbf{D E})\end{array}$ & Estadístico U & $\mathbf{p}$ \\
\hline $\begin{array}{c}\text { Coherencia central } \\
\text { FCR Índice orden de cons- } \\
\text { trucción }\end{array}$ & $1.7(.7)$ & $2.2(.6)$ & 87.5 & .049 \\
FCR Índice estilo & $1.1(.4)$ & $1.5(.3)$ & 66.5 & .006 \\
FCR Índice coherencia & $1.1(.4)$ & $1.4(.3)$ & 72.0 & .012 \\
Flexibilidad mental & & & & \\
Stroop color palabra & $42.4(12.1)$ & $41.4(7.7)$ & 144.0 & $\mathrm{~ns}$ \\
Stroop interferencia & $1.8(8.9)$ & $-.6(6.3)$ & 125.0 & $\mathrm{~ns}$ \\
$\quad$ TMTB & $77.7(31.5)$ & $70.1(20.3)$ & 126.0 & $\mathrm{~ns}$ \\
Fluencia fonológica & $12.8(4.9)$ & $16.5(4.8)$ & 84.0 & .038
\end{tabular}

Nota: AN: Anorexia nerviosa; CS: controles sanos; FCR: Figura Compleja de Rey; TMT: Trail Making Test; ns: no significativo.

\section{Correlaciones entre el desempeño cognitivo y el perfil clínico- psicopatológico}

Se observaron relaciones estadísticamente significativas en el grupo F-AN entre el desempeño en el test de Stroop Color-Palabra y Stroop Interferencia con síntomas de depresión, medidos por el BDI $(\rho=-.58 ; p=.017 \mathrm{y}$ $\rho=-.59 ; p=.015$, respectivamente) y de trastornos alimentarios, valorados por el EDI-II puntuación total $(\rho=-.59 ; \mathrm{p}=.015$ y $\rho=-.52$; $\mathrm{p}=.037$, respectivamente).

\section{Discusión}

El objetivo del presente estudio fue evaluar la coherencia central y la flexibilidad mental en los familiares de primer grado no afectados de pacientes con anorexia nerviosa. La hipótesis del trabajo fue que los familiares no afectados presentarían ineficiencias en estos dominios cognitivos, similares a las previamente observadas en los pacientes (Lang et al., 2014; López et al., 2008; Roberts et al., 2007; Tchanturia et al., 2012). Los resultados de este estudio sostienen parcialmente esta hipótesis.

Se observó que el grupo F-AN presentó 
dificultades en el procesamiento global de la información, evaluadas a partir del desempeño en la copia de la Figura Compleja de Rey (Rey, 2003), caracterizando una debilidad en la coherencia central. Este estilo cognitivo es similar a lo que se ha ampliamente reportado en los pacientes con AN en la fase aguda, así como en individuos recuperados de la enfermedad (Lang et al., 2014; Lopez et al., 2008; Lopez et al., 2009). Con relación a la flexibilidad mental, la única diferencia observada entre los grupos fue en la tarea de fluencia fonológica. En las demás tareas, el grupo F-AN presentó un desempeño similar a los participantes controles sanos.

Los resultados de este trabajo tienen concordancia con la mayor parte de la literatura previa en la que se ha observado que la debilidad en la coherencia central también se encuentra presente en los familiares de primer grado no afectados de los pacientes con AN (Kanakam et al., 2013; Roberts, Tchanturia y Treasure, 2013; Tenconi et al., 2010). Estos resultados, sumados a los previamente mencionados, que han reportado la presencia de este perfil en los pacientes en la fase aguda y luego de la recuperación, apoyan a la hipótesis de que la debilidad en la CC podría ser considerada un endofenotipo neuropsicológico para la anorexia nerviosa.

Con relación a la flexibilidad mental, al contrario de lo esperado, se ha encontrado poca evidencia de que este marcador cognitivo pueda constituirse como un candidato a un endofenotipo neuropsicológico dado que, en la mayoría de las tareas que valoran este dominio, el grupo F-AN presentó un desempeño similar a los CS. La única diferencia observada entre los grupos fue en la tarea de fluencia fonológica. Estos resultados divergen de la mayoría de los trabajos previos que han observado dificultades en la flexibilidad mental en los familiares no afectados de pacientes con AN (Galimberti et al., 2013; Kanakam et al., 2013; Lang, Treasure y Tchanturia, 2015c; Roberts, Tchanturia y Treasure, 2010; Tenconi et al., 2010), pero están en concordancia con los resultados de Brown et al. (2018), que no observaron evidencias de la flexibilidad mental como endofenotipo. La divergencia entre los estudios destaca la necesidad de que se realicen más investigaciones sobre este aspecto neuropsicológico y su potencial rol como endofenotipo para la AN. También es importante mencionar que se ha observado en la literatura una gran heterogeneidad en cuanto a las tareas utilizadas para valorar este dominio cognitivo, lo que, además de limitar la comparación entre los trabajos, podría explicar parcialmente la discrepancia observada entre los estudios.

En cuanto al análisis de las posibles relaciones entre el funcionamiento cognitivo y el perfil clínico y psicopatológico en el grupo F-AN, no se observaron asociaciones entre el desempeño en los índices de coherencia central y el perfil clínico y psicopatológico. En las medidas de la flexibilidad mental, la única relación significativa encontrada fue entre el test de Stroop y la sintomatología depresiva y relacionada con los trastornos alimentarios. Dicha relación carece de relevancia en los resultados del estudio considerando que no se observaron diferencias en el desempeño en el test de Stroop entre los grupos estudiados. Asimismo es válido mencionar que no se observaron diferencias entre los grupos en cuanto al perfil clínico-psicopatológico, y los niveles promedios de síntomas de depresión y relacionados con los trastornos alimentarios estuvieron por debajo de los puntos de corte y de significancia clínica sugeridos en los cuestionarios utilizados.

Un aspecto relevante a ser destacado en la búsqueda de endofenotipos cognitivos es que los trabajos realizados con los pacientes recuperados son de naturaleza trasversal. Esto impide que se obtenga una certeza en cuanto a la estabilidad de los déficits, que es un criterio central a ser cumplido para que un marcador sea clasificado como endofenotipo, ya que no permite saber cómo estaba el funcionamiento cognitivo en el mismo individuo durante y después de la enfermedad. En este sentido, 
se necesitan trabajos que empleen un diseño longitudinal para verificar si las dificultades cognitivas observadas en la etapa aguda de la enfermedad persisten luego de la recuperación completa, considerando tanto la recuperación física como la psicológica (Micali y Dahlgren, 2016). A pesar de esta limitación en relación con los estudios con individuos recuperados, trabajos realizados con adolescentes con AN (Wang et al., 2019), que por definición tienden a presentar una duración más corta de la enfermedad, han encontrado la presencia de déficits en esas funciones cognitivas, aunque de forma atenuada, lo que apoya la evidencia de que puedan representar rasgos endofenotípicos más que cicatrices provenientes de una duración crónica de la enfermedad. En esta misma dirección, Lang et al. (2015) compararon estilos cognitivos entre adolescentes con AN y controles sanos, y encontraron un patrón de rigidez cognitiva significativamente aumentada y una mayor atención hacia los detalles entre los adolescentes con anorexia nerviosa.

Como conclusión, los resultados del presente trabajo, tomados en conjunto con la bibliografía previamente publicada sobre el perfil cognitivo de la AN (investigaciones con pacientes en la fase aguda, individuos recuperados y en pacientes infanto-juveniles), indican que la debilidad en la coherencia central podría ser un candidato a endofenotipo cognitivo para la anorexia nerviosa y aportan poca evidencia sobre la flexibilidad mental como endofenotipo. Estos hallazgos coinciden con lo visto muy frecuentemente en la clínica de los trastornos alimentarios, que es la presencia de rasgos o sintomatología subsindrómica en los familiares de los pacientes. Esta es una de las bases teórico-prácticas para el desarrollo de terapias que incluyen al grupo familiar. Estos rasgos cognitivos, al tener frecuentemente un carácter subclínico en los familiares de primer grado, pueden pasar desapercibidos y son motivo de alteraciones en la dinámica familiar que pueden llegar a favorecer el desarrollo de un trastorno alimentario en un indi- viduo con rasgos predisponentes.

Es por esto que la utilidad práctica de este hallazgo radica en que en un esquema integral del tratamiento de la AN es muy importante no solo realizar el tratamiento psicoterapéutico individual y familiar, sino también evaluar si estos rasgos cognitivos subclínicos pueden estar interfiriendo con la normal dinámica familiar.

De esta forma, los resultados del estudio refuerzan la importancia de los tratamientos de remediación cognitiva (Tchanturia, Lloyd y Lang, 2013) no solo para los pacientes con $\mathrm{AN}$, sino que también remarcan que podrían ser de utilidad para los familiares no afectados (Galimberti et al., 2013). Teniendo en cuenta que la intervención familiar es una herramienta ampliamente utilizada en el tratamiento psicológico de la anorexia, mejorar la percepción del paciente y de sus familiares sobre los sesgos cognitivos podría contribuir a generar conciencia de enfermedad, algo que los pacientes con AN no suelen tener, y generar un impacto positivo en la respuesta al tratamiento como un todo. De hecho, los resultados de un estudio exploratorio (Lang, Treasure y Tchanturia, 2015a) indicaron que involucrar a los miembros de la familia en la terapia de remediación cognitiva produjo beneficios tanto para los pacientes como para sus cuidadores, generando una reducción del estrés y de los niveles de sobrecarga del cuidador, así como mejores capacidades de afrontamiento de la enfermedad.

Finalmente, cabe mencionar algunas limitaciones del trabajo. La primera es el pequeño tamaño de la muestra, que limita la generalización de los resultados obtenidos. Por otro lado, no se analizó en qué medida algunas variables clínicas de los pacientes con anorexia nerviosa (por ejemplo, la edad de inicio y el tiempo de duración de la enfermedad) pudieron afectar la cognición de sus familiares. El funcionamiento cognitivo de los familiares podría verse afectado por el sufrimiento de convivir con un individuo con una enfermedad de larga duración y por el estrés 
de la sobrecarga del cuidador. Si bien este tipo de análisis no era parte de los objetivos del presente estudio, es interesante que sea considerado en investigaciones futuras. También es importante remarcar que no se utilizaron medidas de coherencia central en las que el foco en el detalle sea beneficioso, como el test diseño con cubos (Wechsler, 1999). Se recomienda que futuras investigaciones consideren tareas que abarquen los dos aspectos de la coherencia central, tanto la dificultad en el procesamiento global como el superior foco en el detalle y que utilicen muestras más representativas.

A pesar de las limitaciones aquí mencionadas, el presente trabajo representa un avance más en la búsqueda de endofenotipos para la $\mathrm{AN}$, un área de investigación promisora para esta patología.

\section{Referencias}

Agras, W. S., Brandt, H. A., Bulik, C. M., DolanSewell, R., Fairburn, C. G., Halmi, K. A., ... Wilfley, D. E. (2004). Report of the National Institutes of Health Workshop on Overcoming Barriers to Treatment Research in Anorexia Nervosa. International Journal of Eating Disorders, 35(4), 509-521. https://doi. org/10.1002/eat.10261

American Psychiatric Association. (2013). Diagnostic and statistical manual of mental disorders (5th ed.). Washington, DC: American Psychiatric Association (APA).

Arcelus, J., Mitchell, A. J., Wales, J. y Nielsen, S. (2011). Mortality rates in patients with anorexia nervosa and other eating disorders: A meta-analysis of 36 studies. Archives of General Psychiatry, 68(7), 724-731. https:// doi.org/10.1001/archgenpsychiatry.2011.74

Beck, A., Steer, R. y Brown, G. (2006). BDI, Inventario de Depresión de Beck (2nd ed). Buenos Aires: Paidós.

Benton, A. L. (1968). Differential behavioral effects in frontal lobe disease. Neuropsychologia, $6(1), \quad 53-60 . \quad$ https://doi.org/10.1016/00283932(68)90038-9
Booth, R. (2006). Local-Global Processing and Cognitive Style in Autism Spectrum Disorders and Typical Development. King's College London.

Brenlla, M. y Rodríguez, M. (2006). Adaptación Argentina del Inventario de Depresión de Beck-II (BDI-II). In A. Beck, R. Steer, y G. Brown (Eds.), BDI-II. Inventario de Depresión de Beck (pp. 11-37). Buenos Aires: Paidós.

Brown, M., Loeb, K. L., Mcgrath, R. E., Tiersky, L., Zucker, N. y Carlin, A. (2018). Executive functioning and central coherence in anorexia nervosa : Pilot investigation of a neurocognitive endophenotype. European Eat Disorders Review, 1-10. https://doi.org/10.1002/erv.2597 Butman, J., Allegri, R., Harris, P. y Drake, M. (2000). Fluencia verbal en español datos normativos en Argentina. Medicina Buenos Aires, 60, 561-564. https://doi. org/10.22379/24224022148

Butman, J., Arizaga, R. L., Harris, P., Drake, M., Baumann, D., Pascale, A. De, ... Ollari, J. (2001). El " Mini - Mental State Examination " en español . Normas para Buenos Aires. Revista Neurológica Argentina, 26(1), 13-17.

Fairburn, C. G., Sci, F. M., Cooper, Z., Phil, D., Psych, D., Doll, H. A., ... Psych, F. R. C. (2009). Transdiagnostic Cognitive-Behavioral Therapy for Patients With Eating Disorders: A Two-Site Trial With 60-Week Follow-Up. American Journal of Psychiatry, 166(3), 311-319. https://doi.org/10.1176/appi. ajp.2008.08040608.Transdiagnostic

Fernandéz, A., Marino, J. y Alderete, A. (2002). Estandarización y validez conceptual del Test del Trazo en una muestra de adultos argentinos. Revista Neurológica Argentina, 27(83-88).

Foa, E. B., Huppert, J. D., Leiberg, S., Langner, R., Kichic, R., Hajcak, G. y Salkovskis, P. M. (2002). The obsessive-compulsive inventory: Development and validation of a short version. Psychological Assessment, 14(4), 485-496. https://doi.org/10.1037/1040-3590.14.4.485

Folstein, M., Folstein, S. y McHugh, P. (1975). Mini-Mental State A practical method for grading the cognitive state of patients for the clinician. Journal of Psychiatry Research., 
12, 189-198. https://doi.org/10.1016/00223956(75)90026-6

Fuglset, T. S. (2019). Set-shifting, central coherence and decision-making in individuals recovered from anorexia nervosa: A systematic review. Journal of Eating Disorders, 7(1). https://doi.org/10.1186/s40337-019-0251-5

Fullana, M. A., Tortella-Feliu, M., Caseras, X., Andión, Ó., Torrubia, R. y Mataix-Cols, D. (2005). Psychometric properties of the Spanish version of the Obsessive-Compulsive Inventory - Revised in a non-clinical sample. Journal of Anxiety Disorders, 19(8), 893-903. https:// doi.org/10.1016/j.janxdis.2004.10.004

Galimberti, E., Fadda, E., Cavallini, M. C., Martoni, R. M., Erzegovesi, S. y Bellodi, L. (2013). Executive functioning in anorexia nervosa patients and their unaffected relatives. Psychiatry Research, 208(3), 238-244. https:// doi.org/10.1016/j.psychres.2012.10.001

Garner, D. (1998). Manual del EDI 2: Inventario de trastornos de la conducta alimentaria. Madrid: TEA ediciones.

Golden, C. (1964). Stroop. Test de colores y palabras (TEA edicio). Madrid.

Gottesman, I.I.y Gould, T. D. (2003). TheEndophenotype Concept in Psychiatry: Etymology and Strategic Intentions Irving. American Journal of Psychiatry, 160, 636-645. https://doi. org/10.1111/j.1365-4632.2005.02252.x

Harrison, A., Tchanturia, K., Naumann, U. y Treasure, J. (2012). Social emotional functioning and cognitive styles in eating disorders. The British Journal of Clinical Psychology / the British Psychological Society, 51(3), 261-279. $\quad$ https://doi.org/10.1111/j.20448260.2011.02026.x

Herpertz-Dahlmann, B. (2015). Adolescent Eating Disorders: update on definitions, symptomatology, epidemiology, and comorbidity. Child and Adolescent Psychiatry Clinics of NA, 24(1), 177-196. https://doi.org/10.1016/j. chc.2014.08.003

Holliday, J., Tchanturia, K., Landau, S., Collier, D. y Treasure, J. (2005). Is impaired set-shifting an endophenotype of anorexia nervosa? The American Journal of Psychiatry, 162(12),
2269-2275. https://doi.org/10.1176/appi. ajp.162.12.2269

Jáuregui-lobera, I. (2013). Neuropsychology of eating disorders : 1995 - 2012. Neuropsychiatric Disease and Treatment, 9, 415-430.

Kanakam, N., Raoult, C., Collier, D. y Treasure, J. (2013). Set shifting and central coherence as neurocognitive endophenotypes in eating disorders: A preliminary investigation in twins, (August 2011), 464-475. https://doi.org/ 10.3109/15622975.2012.665478

Kaye, W. H., Wierenga, C. E., Bailer, U. F., Simmons, A. N. y Bischoff-Grethe, A. (2013). Nothing tastes as good as skinny feels: The neurobiology of anorexia nervosa. Trends in Neurosciences, 36(2), 110-120. https://doi. org/10.1016/j.tins.2013.01.003

Lang, K., Lloyd, S., Khondoker, M., Simic, M., Treasure, J. y Tchanturia, K. (2015). Do children and adolescents with anorexia nervosa display an inefficient cognitive processing style? PLoS ONE, 10(7), 1-13. https://doi. org/10.1371/journal.pone.0131724

Lang, K., Lopez, C., Stahl, D., Tchanturia, K. y Treasure, J. (2014). Central coherence in eating disorders: An updated systematic review and meta-analysis. World Journal of Biological Psychiatry, 15(8), 586-598. https://doi.org/10. 3109/15622975.2014.909606

Lang, K., Treasure, J. y Tchanturia, K. (2015a). Acceptability and feasibility of self-help Cognitive Remediation Therapy For Anorexia Nervosa delivered in collaboration with carers: A qualitative preliminary evaluation study. Psychiatry Research, 225(3), 387-394. https:// doi.org/10.1016/j.psychres.2014.12.008

Lang, K., Treasure, J. y Tchanturia, K. (2015b). Is inefficient cognitive processing in anorexia nervosa a familial trait? A neuropsychological pilot study of mothers of offspring with a diagnosis of anorexia nervosa. The World Journal of Biological Psychiatry, 2975, 0-8. https:// doi.org/10.3109/15622975.2015.1112035

Lang, K., Treasure, J. y Tchanturia, K. (2015c). Is inefficient cognitive processing in anorexia nervosa a familial trait? A neuropsychological pilot study of mothers of offspring with a diag- 
nosis of anorexia nervosa, 2975(April 2016), 0-8. https://doi.org/10.3109/15622975.2015.1 112035

Leibovich de Figueroa, N. (1991). Ansiedad: Algunas concepciones teóricas y su evaluación. En M. Casullo, N. Leibovich de Figueroa y M. Aszkenazi (Eds.), Teoría y técnicas de evaluación psicológica. Buenos Aires: Psicoteca Editorial.

Lock, J., Le Grange, D., Agras, W. S., Moye, A., Bryson, S. W. y Booil, J. (2010). Randomized clinical trial comparing family-based treatment to adolescents h anorexia nervosa. Arch Gen Psychiatry, 67(10), 1025-1032. https:// doi.org/10.1001/archgenpsychiatry.2010.128. RANDOMIZED

Lopez, C., Tchanturia, K., Stahl, D., Booth, R., Holliday, J. y Treasure, J. (2008). An Examination of the Concept of Central Coherence in Women with Anorexia Nervosa. International Journal of Eating Disorders, 41(2), 143-152. https://doi.org/10.1002/eat

Lopez, C, Tchanturia, K., Stahl, D. y Treasure, J. (2008). Central coherence in eating disorders: a systematic review. Psychological Medicine, 38(10), 1393-1404. https://doi.org/10.1017/ S0033291708003486

Lopez, C., Tchanturia, K., Stahl, D. y Treasure, J. (2009). Weak central coherence in eating disorders: a step towards looking for an endophenotype of eating disorders. Journal of Clinical and Experimental Neuropsychology, 31(1), 117-125. https://doi. org/10.1080/13803390802036092

Lounes, N., Khan, G. y Tchanturia, K. (2011). Assessment of cognitive flexibility in anorexia nervosa--self-report or experimental measure? A brief report. Journal of the International Neuropsychological Society: JINS, 17(5), 925-928. https://doi.org/10.1017/ S1355617711000671

Micali, N. y Dahlgren, C. L. (2016). All that glisters is not an endophenotype : rethinking endophenotypes in anorexia nervosa. European Child y Adolescent Psychiatry, 25(11), 1149-1150. https://doi.org/10.1007/s00787016-0910-x
Miranda, A. L., Jaramillo, C., Valencia, J. y Duque, J. (2003). El uso de endofenotipos en el estudio del componente genético de la esquizofrenia. Revista Colombiana de Psiquiatría, 32(3), 237-248.

Pike, K. M. (1998). Long-term course of anorexia nervosa: Response, relapse, remission, and recovery. Clinical Psychology Review, 18(4), 447-475. https://doi.org/10.1016/S02727358(98)00014-2

Reitan, R. M. (1958). Validity of the trail making test as an indicator of organic brain damage. Perceptual and Motor Skills, 8, 271-276.

Rey, A. (2003). Test de copia y de reproducción de memoria de figuras geométricas complejas. Madrid: TEA ediciones.

Roberts, M. E., Tchanturia, K., Stahl, D., Southgate, L. y Treasure, J. (2007). A systematic review and meta-analysis of set-shifting ability in eating disorders. Psychological Medicine, 37(8), 1075-1084. https://doi.org/10.1017/ S0033291707009877

Roberts, M. E., Tchanturia, K. y Treasure, J. L. (2010). Exploring the neurocognitive signature of poor set-shifting in anorexia and bulimia nervosa. Journal of Psychiatric Research, 44(14), 964-970. https://doi.org/10.1016/j. jpsychires.2010.03.001

Roberts, M. E., Tchanturia, K. y Treasure, J. L. (2013). Is attention to detail a similarly strong candidate endophenotype for anorexia nervosa and bulimia nervosa? The World Journal of Biological Psychiatry, 14(October 2011), 452-463. https://doi.org/10.3109/15622975.2 011.639804

Rutsztein, G., Murawski, B., Elizathe, L. y Scappatura, L. (2010). Trastornos alimentarios: Detección en adolescentes mujeres y varones de Buenos Aires. Un estudio de doble fase. Revista Mexicana de Trastornos Alimentarios, 1, 48-61.

Spielberger, C., Gorsuc, R. y Lushene, R. (1982). STAI: Cuestionario de ansiedad estado-rasgo. Versión española adaptada. Madrid: TEA ediciones.

Talbot, A., Hay, P., Buckett, G. y Touyz, S. (2015). Cognitive deficits as an endophenotype for 
anorexia nervosa: An accepted fact or a need for re-examination? International Journal of Eating Disorders, 48(1), 15-25. https://doi. org/10.1002/eat.22332

Tapajóz, F., Catoira, N., Soneira, S., Aulicino, A., Calandri, I. y Allegri, R. F. (2016). Estudio de las funciones cognitivas en pacientes con trastornos alimentarios. Revista Neuropsicologia Latinoamericana, 8(3), 42-51. https://doi. org/10.5579/rnl.2016.0300

Tapajóz P. de Sampaio, F., Soneira, S., Aulicino, A., Martese, G., Iturry, M. y Allegri, R. F. (2013). Theory of mind and central coherence in eating disorders: Two sides of the same coin? Psychiatry Research, 210, 1116-1122. https://doi.org/10.1016/j.psychres.2013.08.051

Tchanturia, K., Davies, H., Roberts, M., Harrison, A., Nakazato, M., Schmidt, U., ... Morris, R. (2012). Poor cognitive flexibility in eating disorders: examining the evidence using the Wisconsin Card Sorting Task. PloS One, 7(1), 1-5. https://doi.org/10.1371/journal. pone. 0028331

Tchanturia, K., Harrison, A., Davies, H., Roberts, M., Oldershaw, A., Nakazato, M., ... Treasure, J. (2011). Cognitive flexibility and clinical severity in eating disorders. PloS One, 6(6), 1-5. https://doi.org/10.1371/journal. pone. 0020462

Tchanturia, K., Lloyd, S. y Lang, K. (2013). Cognitive remediation therapy for anorexia nervosa: current evidence and future research directions. The International Journal of Eating Disorders, 46(5), 492-495. https://doi. org/10.1002/eat.22106

Tenconi, E., Santonastaso, P., Degortes, D.,
Bosello, R., Titton, F., Mapelli, D. y Favaro, A. (2010). Set-shifting abilities, central coherence, and handedness in anorexia nervosa patients, their unaffected siblings and healthy controls: Exploring putative endophenotypes. The World Journal of Biological Psychiatry, 11(November 2009), 813-823. https://doi.org/ $10.3109 / 15622975.2010 .483250$

Treasure, J. L. (2007). Getting Beneath the Phenotype ofAnorexia Nervosa: The Search for Viable Endophenotypes and Genotypes. The Canadian Journal of Psychiatry, 52(4), 212-219. https://doi.org/10.1177/070674370705200402

Treasure, J., Zipfel, S., Micali, N., Wade, T., Stice, E., Claudino, A., ... Wentz, E. (2015). Anorexia nervosa. Nature Reviews Disease Primers, 1(November), 1-22. https://doi.org/10.1038/ $\operatorname{nrdp} .2015 .74$

Waller, G., Gray, E., Hinrichsen, H., Mountford, V., Lawson, R. y Patient, E. (2014). Cognitive-behavioral therapy for bulimia nervosa and atypical bulimic nervosa: Effectiveness in clinical settings. International Journal of Eating Disorders, 47(1), 13-17. https://doi. org/10.1002/eat.22181

Wang, S. B., Gray, E. K., Coniglio, K. A., Murray, H. B., Becker, K. R., Thomas, J. J., ... Eddy, K. T. (2019). Cognitive rigidity and heightened attention to detail occur transdiagnostically in adolescents with eating disorders. Eating Disorders, 0266, 1-13. https://doi.org/10.1080 /10640266.2019.1656470

Wechsler, D. (1999). WASI: Wechsler Abbreviated Scale of Intelligence. New York: The Psychological Corporation: Harcourt Brace y Company.

Recibido: 11 de mayo de 2020 Aceptado: 15 de septiembre de 2021 